\title{
A Responsive "Nano String Light" for Highly Efficient mRNA Imaging in Living Cells via Accelerated DNA Cascade Reaction
}

Kewei Ren, ${ }^{\dagger}$ Yifan Xu, ${ }^{\dagger}$ Ying Liu, ${ }^{* \dagger}$ Min Yang and Huangxian Ju* ${ }^{\ddagger} \dagger$

†State Key Laboratory of Analytical Chemistry for Life Science, School of Chemistry and Chemical Engineering, Nanjing University, Nanjing, 210023, P.R. China.

Department of Pharmaceutical \& Biological Chemistry, UCL School of Pharmacy, University College London, London WC1N 1AX, UK.

*Address correspondence to yingliu@nju.edu.cn, hxju@nju.edu.cn 
ABSTRACT: Nonenzymatic DNA catalytic amplification strategies have greatly benefited bioanalysis. However, long period incubation is usually required due to its relatively low reaction rate and efficiency, which limits its in vivo application. Here we design a responsive DNA Nano String Light (DNSL) by interval hybridization of modified hairpin DNA probe pairs to a DNA nanowire generated by rolling circle amplification, and realize accelerated DNA cascade reaction (DCR) for fast and highly efficient mRNA imaging in living cells. Target mRNA initiates interval hybridization of two hairpin probes sequentially along the DNA nanowire, and results in instant lighting up of the whole DNA nanowire with high signal gain due to the fast opening of all the self-quenched hairpins. The reaction time is about 6.7 times shorter compared with regular DNA cascade reaction due to the acceleration based on domino effect. The cell delivery is achieved by modifying one of the hairpin probes with folic acid, and this intracellular imaging strategy is verified using human HeLa cells and intracellular survivin mRNA with series of suppressed expressions as model, which provides a useful platform for fast and highly efficient detection of low-abundance nucleic acids in living cells.

KEYWORDS: nucleic acid amplification, DNA cascade reaction, DNA nano string light, mRNA imaging, confined space 
Visualization of biomolecules in living cells remains challenging owing to their low expression levels and the complex intracellular environment. Although amplification techniques based on nucleic acid cascade reaction have benefited ultrasensitive biosensing of various targets such as RNA, proteins, and small molecule biomarkers in many areas, including molecular diagnosis and digital circuit computation, ${ }^{1-4}$ most amplification procedures, such as polymerase chain reaction $(\mathrm{PCR})^{5}$ and rolling circle amplification (RCA), ${ }^{6,7}$ require the participation of exotic enzymes, which hampers the further application of these techniques inside living cells. ${ }^{8}$ Nonenzymatic catalytic amplification strategies based on DNA cascade reaction, such as hybridization chain reaction $(\mathrm{HCR})$ and catalytic hairpin assembly $(\mathrm{CHA}),{ }^{9-11}$ are independent of exotic enzymes during the reaction process, therefore have become valuable tools for biomolecule detection both in vitro and in vivo. ${ }^{12-14}$ Target initiated HCR has been programmed on gold nanoparticle ${ }^{15}$ and graphene oxide ${ }^{16}$ for messenger RNA (mRNA) and microRNA imaging in living cells. However, the kinetics of HCR depends on the diffusion of DNA reactants for random collision and interaction in the homogeneous environment. ${ }^{15,16}$ The reaction proceeding requires continuously searching for the next hybridizing probe in a three-dimensional fluidic space, which greatly prolongs the reaction time and compromises the reaction efficiency. ${ }^{17-19}$ The amplification of 2-5 fold/hour is usually achieved for conventional HCR in homogeneous environment, ${ }^{20}$ and the incubation time for sufficient reaction is often more than 2 hours. ${ }^{15,16}$ Therefore, reaction acceleration is urgently needed for HCR in situ application. 
Confining successive reactants together in a compact space maintains high local concentrations of reagents, thus promotes substrates transportation, protects them against damage, and accelerates reaction. ${ }^{21-24}$ DNA origami platforms have been used to transport enzymatic reaction substrates ${ }^{25}$ and DNA probes in heterogeneous hybridizations $^{26-28}$ and strand displacement reaction ${ }^{29}$ for reaction acceleration. However, the related works all focus on studying reaction mechanism up to now, and haven't been extended to practical application for biosensing and bioimaging due to the limited number of imaging probes involved in successive reaction process and corresponding low sensitivity in detection. ${ }^{26}$

Here we designed a DNA "nano string light" (DNSL) responsive to target mRNA based on accelerated DNA cascade reaction (DCR) along a DNA nanowire, and achieved sensitive mRNA imaging in living cells with highly enhanced reaction rate and efficiency. The DNSL was constructed by interval hybridization of modified DNA hairpin probe pairs ( $\mathrm{H} 1$ and $\mathrm{H} 2)$ to a DNA nanowire with reduplicated sequence segments generated by rolling circle amplification (RCA) (Scheme 1a). Self-quenched H1 was labelled with folic acid (FA) for receptor-mediated human cervix carcinoma (HeLa) cell endocytosis of DNSL. The intracellular target survivin mRNA then hybridized with one $\mathrm{H} 1$ in DNSL to trigger the cascade hybridization of $\mathrm{H} 1$ and $\mathrm{H} 2$ along the DNA nanowire due to their alternate arrangement in DNSL and programmed distance, which could instantly light up the whole DNSL with highly amplified signal gain (Scheme 1b). Compared with previously reported nonenzymatic catalytic amplification techniques $^{30,31}$ and successive DNA hybridization on nanoplatform, ${ }^{27-29}$ 
the DNSL contained numbers of $\mathrm{H} 1$ and $\mathrm{H} 2$ as successive reactants in a confined space, which not only accelerated the reaction with high efficiency, but also enhanced the detection sensitivity with high signal gain. The biocompatible DNSL as a delivery vehicle also facilitated the intracellular delivery without the usage of exotic transfection reagents. Therefore, it created a promising detection platform for intracellular imaging and possesses potential application for disease diagnostics and therapy.

\section{RESULTS AND DISCUSSION}

Synthesis and Characterization of DNSL. As it shown in Scheme 1a, H1 and H2 were alternately aligned on the long continuous DNA nanowire produced by RCA to form DNSL. The H1 was synthesized by hybridization of hairpin 1 with $\mathrm{H} 1$ connect. Hairpin 1 was composed of linkage sequence L1, toehold T1, and hairpin structure ST2'S', which was self-quenched by labeling with 5-carboxyfluorescein (FAM) dye and its black hole quencher (BHQ1) and recovered fluorescence upon hairpin opening (Table S1), while H1 connect was composed of sequence A1 which anchored to DNA nanowire and sequence L1' which was complementary to L1 at the 5'-end of hairpin 1. H2 was a hairpin structure (S'T1'S) tailed with toehold T2, linkage sequence L2 and anchoring sequence A2 at 3' end (Scheme 1a). Toehold T1 in hairpin 1 was complementary to $\mathrm{T} 1$ ' in $\mathrm{H} 2$, and toehold $\mathrm{T} 2$ in $\mathrm{H} 2$ was complementary to $\mathrm{T} 2$ ' in hairpin 1. The sequence of target mRNA was complementary to the sequence of toehold T1 and $\mathrm{S}$ in hairpin 1 to trigger the accelerated DCR along the nanowire. The synthesis of DNSL was confirmed by $0.6 \%$ agarose gel electrophoresis experiment. After RCA reaction, a band with bases ranging from 400 to 1200 was appeared in lane 1 (Figure 
1a), suggesting the formation of DNA nanowire. ${ }^{32}$ After DNA nanowire was mixed with H1 (lane 2, Figure 1a) and H2 (lane 3, Figure 1a), both the bands for H1 and H2 disappeared and an extended band with lower mobility was observed (lane 4, Figure 1a), indicating the successful formation of DNSL. The DNSL structure was also characterized by atomic force microscopy (AFM). The DNSL tubes appeared to be quite rigid and monodisperse with an average length of $260 \pm 100 \mathrm{~nm}$ and height of 2.5 nm (Figure 1b,c). Given that A1 and A2 were 24 bases individually (about $8.16 \mathrm{~nm}$ ), ${ }^{33}$ each DNSL contained $15 \pm 6$ pairs of $\mathrm{H} 1 / \mathrm{H} 2$. To further confirm the number of $\mathrm{H} 1$ and $\mathrm{H} 2$ in DNSL, aminomethylcoumarin (AMCA) labelled H0 was introduced and anchored at the tip of nanowire, which was subsequently hybridized with 5carboxyfluorescein (FAM) labelled H1 and tetramethylrhodamine (TAMRA) labelled H2 to synthesize a tricolour DNSL. Based on the standard calibration curves of AMCAH0, FAM-hairpin1 and TAMRA-H2 (Figure S1), the amount of FAM and TAMRA were about 15.6 and 15.8 times the amount of AMCA respectively, indicating that each DNSL contained about 16 pairs of $\mathrm{H} 1 / \mathrm{H} 2$, consistent with the AFM result. Compared with the previous reported DNA successive hybridization in DNA nanostructures, ${ }^{27-29}$ here much more number of reactant DNA probes were involved in the DNSL due to their compact alignment, which contributed to the signal amplification and benefited intracellular imaging.

The serum stability of DNSL was evaluated by measuring the fluorescence recovery of FAM from self-quenched H1 in DNSL upon treatment with 10\% FBS reaction buffer over $12 \mathrm{~h}$, which was much less than the control couple of free H1 (Figure S2), 
indicating that DNSL structure could protect $\mathrm{H} 1$ from nuclease degradation during intracellular delivery.

Feasibility of Target mRNA Triggered HCR and DCR. The feasibility of target mRNA triggered HCR was firstly verified in homogeneous solution with free $\mathrm{H} 1$ and $\mathrm{H} 2$ via $8 \%$ PAGE analysis (Figure S3a). After incubating survivin mRNA with the mixture of $\mathrm{H} 1$ and $\mathrm{H} 2$, the bands representing $\mathrm{H} 1$ and $\mathrm{H} 2$ disappeared with a new ladder shaped band appeared (lanes 4), indicating the successful proceeding of HCR in homogeneous solution. Incubating $\mathrm{H} 1$ and $\mathrm{H} 2$ mixture solution with control mRNA with scrambled sequence did not yield any band of larger molecular mass (lanes 5), demonstrating the high specificity of survivin mRNA triggered HCR. To prove the accelerated DCR along the DNA nanowire, fluorescence resonance energy transfer (FRET) experiment was designed in the bicolor DNSL which was assembled with fluorescence donor FAM labelled H1, fluorescence acceptor TAMRA labelled H2 and DNA nanowire. The bicolor DNSL showed only FAM fluorescence in the absence of survivin mRNA, and demonstrated FRET signal proportional to mRNA concentration due to the opening of hairpin 1 and 2 and corresponding close proximity between FAM and TAMRA (Figure S3b). To compare the accelerated DCR in DNSL with the HCR in homogeneous solution, $100 \mathrm{nM} \mathrm{H} 1$ was mixed with $100 \mathrm{nM} \mathrm{H} 2$ in the presence and absence of DNA nanowire respectively. After the mixtures were centrifuged by ultrafiltration purification column with the cut-off molecular mass of $100 \mathrm{KD}, 10 \mathrm{nM}$ survivin mRNA was added to the concentrate and filtrate solutions respectively. ${ }^{34}$ Substantial fluorescence was observed from the concentrate in the presence of nanowire 
(Figure S3c), indicating the generation of DNSL and the successful proceeding of DCR in DNSL. On the contrary, most fluorescence signal was collected from the filtrate in the absence of DNA nanowire (Figure S3d), since free H1 and H2 remained in homogeneous solution where the HCR was initiated. The stronger fluorescence intensity from the DNSL concentrate demonstrated the higher reaction efficiency of DCR in confined DNSL nanostructure.

To prove the proceeding of cascade $\mathrm{H} 1 / \mathrm{H} 2$ hybridization in DNSL, control DNSL (cDNSL) was prepared by assembling $\mathrm{H} 1$ and $\mathrm{H} 4$ on DNA nanowire. Although survivin mRNA could hybridize with $\mathrm{H} 1$, no cascade hybridization proceeding along cDNSL was observed due to the non-complementary of $\mathrm{H} 1$ and $\mathrm{H} 4$. Upon addition of $5 \mathrm{nM}$ survivin mRNA, the FAM fluorescence recovery from self-quenched H1 in DNSL was about 13.3 times that in cDNSL (Figure S4), which indicated that 16 pairs of $\mathrm{H} 1 / \mathrm{H} 2$ contained in DNSL were almost opened to recover the fluorescence due to the cascade hybridization of $\mathrm{H} 1$ and $\mathrm{H} 2$.

Kinetic Analysis of Accelerated DCR in DNSL. Time-dependent fluorescence analysis was carried out to study the reaction kinetics of both DCR in DNSL and HCR in homogeneous solution. The FAM fluorescence recovery from self-quenched H1 was measured for $2 \mathrm{~h}$ in response to $10 \mathrm{nM}$ survivin mRNA activation. Upon addition of survivin mRNA to DNSL, it showed a substantial fluorescence increase for timedependent fluorescence spectra (Figure 2a, pink line). Compared with the HCR in homogeneous solution (Figure 2a, red line), DCR resulted in large fluorescence enhancement with substantial acceleration. Fluorescence signal was barely seen from 
both systems in the absence of survivin mRNA. To demonstrate the speed-up effect of DCR, the fluorescence intensities for DCR and HCR in Figure 2a were normalized. The completion time for DCR was about 15 min (Figure S5a), which is 6.7 times shorter than that of HCR (Figure S5b). The latter usually requires over-hours incubation time in normal signal amplification strategies. Thus the DCR impressively shortened the reaction time and highly facilitated the detection process.

To further clarify the high reaction rate and efficiency for DCR, the collision theory was brought to analyze the reaction process. The collision frequency is proportional to the reactants concentrations. ${ }^{35}$ From $V=1 / c \mathrm{~N}$ (where $c$ is $\mathrm{H} 1(\mathrm{H} 2)$ concentration, and $\mathrm{N}$ is Avogadro constant), the volume of a sphere containing both $\mathrm{H} 1$ and $\mathrm{H} 2$ molecules was calculated to be $1.7 \times 10^{-17} \mathrm{~L}$ with a radius of $157 \mathrm{~nm}$ in a homogeneous solution containing $100 \mathrm{nM} \mathrm{H} 1$ and $\mathrm{H} 2$ (Figure 2b). In the situation of DNSL, the distance between $\mathrm{H} 1$ and $\mathrm{H} 2$ was about $24.4 \mathrm{~nm}$ including the anchoring segment and linkage segments of $\mathrm{H} 1$ and $\mathrm{H} 2$ (72 base pairs). Confined within a sphere of $24.4 \mathrm{~nm}$ in radius, the local concentrations of $\mathrm{H} 1$ and $\mathrm{H} 2$ in DNSL were calculated as $27.3 \mu \mathrm{M}$. The local concentrations of $\mathrm{H} 1$ and $\mathrm{H} 2$ in DNSL increased by 273 folds as compared with the situation in homogeneous solution. The increase in local concentration highly enhanced the collision frequency between $\mathrm{H} 1$ and $\mathrm{H} 2$ in DNSL, resulting in the accelerated reaction and enhanced reaction efficiency.

In Vitro Response to survivin mRNA. The amplification efficiency of DCR (Figure 3a) was firstly compared to those of HCR (Figure 3d), and mRNA-H1 reaction without amplification (H1R) (Figure 3g) through in vitro detection of target survivin mRNA by 
measuring the FAM fluorescence recovery from self-quenched H1 upon 15-min incubation. The incubation time was substantially shortened here since DCR was highly accelerated via domino effect compared with HCR in homogeneous solution. The DCR led to much stronger fluorescence intensity with the increasing survivin mRNA concentration (Figure 3b). The fluorescence could be clearly observed from $10.0 \mathrm{pM}$ survivin mRNA after DCR amplification, which was 20.3 times that from HCR amplification (Figure 3e) and 28.6 times that from H1R (Figure 3h) respectively, demonstrating the high signal amplification efficiency of DCR. A quasi-linear relationship between the logarithmic value of fluorescence intensity and logarithmic value of survivin mRNA concentration was obtained in the range from $50.0 \mathrm{pM}$ to 50.0 $\mathrm{nM}$ with a limit of detection (LOD) of $10.9 \mathrm{pM}$ (Figure 3c). The LOD was determined by extrapolating the concentration from the signal equal to background signal plus 3SD of the background signal. In contrast, there was no discriminable fluorescence signal compared with background at survivin mRNA concentrations below $1.0 \mathrm{nM}$ for HCR (Figure 3e) and $5.0 \mathrm{nM}$ for H1R (Figure 3h). The detectable linear ranges were 1.0 to 100.0 $\mathrm{nM}$ for HCR with LOD at $566.8 \mathrm{pM}$ (Figure 3f) and 5.0 to $100.0 \mathrm{nM}$ for H1R with LOD at $3528.6 \mathrm{pM}$ (Figure 3i) respectively. The LOD for DCR was about 52.2fold lower than that of HCR, and 323.7-fold lower than that of H1R.

The specificity of the proposed strategy was also investigated with the control mRNA with scrambled sequence, single-base mismatched mRNA and three-bases mismatched mRNA. By reacting with DNSL, control mRNA, single-base mismatched mRNA, and three-base mismatched mRNA all showed very low fluorescence responses, close to 
that of the blank control (Figure S6). The fluorescence intensity was 518.7 in response to $20.0 \mathrm{nM}$ survivin mRNA triggered DCR, which is about 7.1 times that of single-base mismatched mRNA, 7.6 times that of three-base mismatched mRNA, and 7.8 times that of control mRNA at the same concentration, demonstrating the excellent specificity of the DNSL with DCR acceleration and amplification.

Live Cell Imaging of survivin mRNA via DCR. The feasibility of the DCR strategy for in situ visualizing intracellular survivin mRNA was explored with HeLa cells as a model. The FAM fluorescence recovery from self-quenched H1 in DNSL in response to survivin mRNA was monitored in living cells, which gradually increased according to incubation time and reached saturation at $2 \mathrm{~h}$ (Figure S7), therefore 2-h incubation was used for intracellular imaging. Compared with the previous reports which require over $5 \mathrm{~h}$ of incubation, ${ }^{16,30}$ the detection period for in vivo imaging was substantially shortened due to the fast uptake and subsequent acceleration effect of DCR, which is quite important for in situ diagnosis. The fluorescence signal from FAM in Z-stack images exhibited a position-sensitive dependence, ${ }^{37}$ demonstrating intracellular localization of DNSL (Figure S8).

To verify the intracellular delivery specificity of DNSL was induced by the folic acid (FA) receptor-mediated cell endocytosis, a series of control experiments were performed and compared with the cellular uptakes of DNSL (Figure S9). The HeLa cells showed strong fluorescence within a 2-h incubation of DNSL ("HeLa, DNSL", Figure S9), whereas the cells incubated with FA-free DNSL displayed little fluorescence signal ("HeLa, FA-free DNSL", Figure S9), the DNSL couldn't get into 
cell in the absence of FA due to its strong negative charge. The internalization of DNSL was also blocked when excessive free FA was co-incubated ("HeLa, FA+DNSL", Figure S9), further proving that FA played important roles in the targeted delivery of the DNSL to HeLa cells. The delivery specificity to HeLa cells was also evaluated by incubating FA receptor-negative human epidermal HaCaT cells with the DNSL ("HaCaT, DNSL", Figure S9), which demonstrated little fluorescence after 2-h incubation, suggesting the precise recognition and specific delivery of DNSL to target HeLa cells.

The specificity of DNSL for the intracellular survivin mRNA imaging was demonstrated by incubating HeLa cells with the control DNSL assembled with selfquenched H11 (co-labelled with FAM and BHQ1) and H2, while H11 is not responsive to survivin mRNA. The control DNSL showed little intracellular fluorescence ("HeLa, Control DNSL”, Figure S9). By treated with 5 nM YM155 (survivin mRNA expression inhibitor), the survivin mRNA expression was completely suppressed in HeLa cells (control HeLa) (Figure S10). ${ }^{38}$ When incubated with DNSL, little fluorescence was observed from control HeLa due to the drastically decrease of intracellular survivin mRNA ("Control HeLa, DNSL", Figure S9), proving the specificity of DNSL response to the target mRNA. In order to perform FRET experiment to further confirm the response specificity of DNSL to intracellular survivin mRNA, bicolor DNSL was synthesized by assembling FAM labelled $\mathrm{H} 1$ and TAMRA labelled $\mathrm{H} 2$, and incubated with HeLa cells. As shown in Figure S11, successful intracellular FRET process was achieved between FAM and TAMRA dyes upon the opening of hairpin structured H1 
and H2 ("Bicolor DNSL"), and only FAM fluorescence was observed when bicolor DNSL was incubated with survivin mRNA expression suppressed control HeLa cells ("Bicolor DNSL, Control HeLa"). The control bicolor DNSL which was not responsive to survivin mRNA was also prepared with FAM-labelled H11 and TAMRA-labelled H2, which didn't demonstrate FRET between FAM and TAMRA dyes either ("Control bicolor DNSL", Figure S11). The intense FRET signal upon the activation of target mRNA for bicolor DNSL in HeLa cells eliminated the false-positive signal from thermodynamic fluctuations and chemical interferences (such as nuclease and glutathione), ${ }^{39}$ and confirmed the successfully intracellular proceeding of DCR. Though relatively large, the DNSL has outstanding biocompatibility due to DNA selfassembled skeleton, and had little influence on cell morphology (Figure S12) and cell viability after 2-h incubation (Figure S13).

To demonstrate the application of this strategy for signal amplification of intracellular mRNA imaging, HeLa cells were incubated with DNSL composed of self-quenched $\mathrm{H} 1$ and $\mathrm{H} 2$ to achieve folic acid (FA) receptor-mediated cell endocytosis, which showed bright fluorescence upon the signal amplification from DCR (Figure 4a). As control, self-quenched $\mathrm{H} 1$ and the mixture of self-quenched $\mathrm{H} 1$ and $\mathrm{H} 2$ were transfected into HeLa cells for intracellular survivin mRNA imaging. Both of them showed much less fluorescence (Figure 4a). The fluorescence intensity from DCR amplification was 11.9 times that of imaging merely with $\mathrm{H} 1$, and 2.85 times that of imaging with $\mathrm{HCR}$ amplification (Figure 4b). These results demonstrated the highly catalytic amplification ability of DCR for intracellular survivin mRNA imaging. 
The potential of the proposed strategy for quantitative evaluation of the relative expression level of survivin mRNA in living HeLa cells was evaluated. A large pool of HeLa cells was treated with YM155 in different concentrations to decrease the intracellular survivin mRNA expression to mimic the natural change of mRNA expression upon biological stimulus. ${ }^{40}$ The fluorescence signals were amplified with DCR and HCR in cytoplasm for comparison. The bright fluorescence was still observed from cells treated with $3.0 \mathrm{nM}$ YM155 for DCR signal amplification, while the cells treated with YM155 at the same concentrations demonstrated indistinguishable fluorescence signals for HCR signal amplification (Figure 5). This result was also confirmed by flow cytometric assay (Figure S14) and RT-PCR (Figure S15). These results suggest that the developed strategy is appropriate for high-sensitive intracellular sensing of the low-abundance nucleic acids.

To illustrate the generality of the proposed strategy, H3 and H4 probes were designed in response to TK1 mRNA and assembled on DNA nanowire to form a new DNA nano string light (TDNSL) for detection of TK1 mRNA. Here H3 was self-quenched by labeling it with TAMRA and BHQ2, and the feasibility of TDNSL was verified by measuring the recovery of TAMRA fluorescence upon H3 opening. After addition of 5 nM TK1 mRNA to 100 nM TDNSL, an intense fluorescence was observed (Figure S16), indicating the successful proceeding of DCR in TDNSL and the high efficiency for TK1 mRNA detection. The simultaneous detection of multiplex mRNAs was also demonstrated by incubating DNSL, TDNSL, the mixture of DNSL and TDNSL with 5 $\mathrm{nM}$ survivin mRNA and $5 \mathrm{nM}$ TK1 mRNA, respectively, to compare their fluorescence 
spectra. The fluorescence of FAM was only observed in the presence of both $s$-mRNA and DNSL, while the TAMRA fluorescence was only observed in the presence of both T-mRNA and TDNSL (Figure S17a). Very low fluorescence for cross reactions was observed, indicating the imaging applicability of the proposed strategy for multiple mRNAs. This applicability was also demonstrated intracellularly by incubating HeLa cells with the mixture of DNSL and TDNSL, both the FAM fluorescence signal for survivin mRNA from DNSL and the TAMRA fluorescence signal for TK1 mRNA from TDNSL were clearly observed in HeLa cells (Figure S17b), indicating the excellent capability of this technique for imaging multiple mRNAs in living cell.

\section{CONCLUSIONS}

We present a concept of accelerated DCR along a DNA nanowire based on a DNSL nanostructure, which leads to fast and highly efficient mRNA quantitative detection in vitro and in situ quantitative evaluation of the relative expression level of mRNA in live cells. The DNSL can be conveniently synthesized via the self-assembly of selfquenched $\mathrm{H} 1$ probe and $\mathrm{H} 2$ along a designed DNA nanowire, and shows excellent specificity for both in vitro detection and in vivo imaging of mRNA. The DCR activated by target mRNA proceeds along the nanowire and instantly lights up the whole DNSL, leading to high response. The quantitative evaluation of intracellular survivin mRNA levels upon drug treatment demonstrates its practicability in intracellular sensing of the low-abundance nucleic acids. Compared with conventional HCR in homogeneous solution, this strategy significantly shortens the incubation time and enhances detection sensitivity. By designing the recognition sequence of T1S and corresponding S'T1', this 
strategy could conveniently be used as a universal platform for the detection of different mRNAs and highly efficient imaging of nucleic acids in live cells.

\section{EXPERIMENTAL SECTION}

Materials and Reagents. Phi29 DNA polymerase, T4 DNA ligase, exonuclease I, exonuclease III and dNTPs were purchased from New England Biolabs Ltd. DNA purification kit was obtained from ComWin Biotech Co., Ltd (China). YM155 and MTT were from Sigma-Aldrich (USA). Phosphate buffer saline (PBS, pH 7.4) contained $136.7 \mathrm{mM} \mathrm{NaCl}, 2.7 \mathrm{mM} \mathrm{KCl}, 8.72 \mathrm{mM} \mathrm{Na}_{2} \mathrm{HPO}_{4}$ and $1.41 \mathrm{mM} \mathrm{KH}_{2} \mathrm{PO}_{4}$. All other reagents were of analytical grade. All aqueous solutions were prepared using ultrapure water ( $\geqslant 18 \mathrm{M} \Omega$, Milli-Q, Millipore). All mRNAs were obtained from GenePharma Co. Ltd. (Shanghai, China) with the sequences as follows: survivin mRNA, 5'-UCUCAAGGACCACCGCAUCUCUAC-3', single mismatched survivin mRNA, 5'-UCUCAAGGACCACCGCAUCACUAC-3', three mismatched survivin mRNA, 5'-UCUCAAGGACCACCGGAUGUCÁAC-3', control mRNA, 5'-GGUGA AACCGCAUCUCUACUAAAGAUA. The mismatched bases were shown in underlined. TK1 mRNA, 5'-UGAGUUUCUGUUCUCCCUGGGAAG-3'. All of the DNAs were synthesized and purified by Sangon Biotech Co., Ltd (Shanghai, China). Their sequences were listed in Table S1.

Apparatus. Absorption spectra were recorded on an UV-3600 UV-Vis-NIR spectrophotometer (Shimadzu Company, Japan). The gel electrophoresis was performed on a DYCP-31BN electrophoresis analyser (Liuyi Instrument Company, China) and imaged on Bio-rad ChemDoc XRS (Bio-Rad, USA). Fluorescence spectra 
were measured on an F-7000 spectrometer (HITACHI, Japan). MTT assay was carried out on Hitachi/Roche System Cobas 6000 (680, Bio-Rad, USA). Confocal fluorescence imaging of cells was performed on a TCS SP5 confocal laser scanning microscope (Leica, Germany). Flow cytometric analysis was performed on a Coulter FC-500 flow cytometer (Beckman-Coulter). Real-time reverse transcription polymerase chain reaction (RT-PCR) were performed on a CFX96 touch RT-PCR detection system (Bio$\mathrm{Rad})$.

Preparation of Circular DNA Template. $4.2 \mu \mathrm{L}$ of $100 \mu \mathrm{M}$ phosphorylated linear DNA and $4.2 \mu \mathrm{L}$ of $100 \mu \mathrm{M}$ ligation DNA were mixed and annealed at $95^{\circ} \mathrm{C}$ for $4 \mathrm{~min}$. After the mixture was slowly cooled to room temperature over $2 \mathrm{~h}, 1 \mu \mathrm{L}$ of T4 DNA ligase $(400 \mathrm{U} / \mu \mathrm{L}), 2 \mu \mathrm{L}$ of $10 \times \mathrm{T} 4 \mathrm{DNA}$ buffer and $8.6 \mu \mathrm{L}$ of ultrapure water were added and the solution was incubated at $25^{\circ} \mathrm{C}$ for $16 \mathrm{~h}$. After T4 DNA ligase was inactivated by heating at $65^{\circ} \mathrm{C}$ for $10 \mathrm{~min}, 4 \mu \mathrm{L}$ of exonuclease $\mathrm{I}(20 \mathrm{U} / \mu \mathrm{L})$ and $4 \mu \mathrm{L}$ of exonuclease III $(100 \mathrm{U} / \mu \mathrm{L})$ were added and incubated at $37{ }^{\circ} \mathrm{C}$ for $8 \mathrm{~h}$ to degrade DNA ligation. After heating at $80^{\circ} \mathrm{C}$ for 15 min to denature the exonuclease I and exonuclease III, the resulting circular DNA template was stored at $4{ }^{\circ} \mathrm{C}$ prior to use.

Preparation of DNA Nanowire. The DNA nanowire was prepared by rolling circle amplification (RCA). ${ }^{41,42} 10 \mu \mathrm{L}$ of $3 \mu \mathrm{M}$ circular DNA template and $0.5 \mu \mathrm{L}$ of $100 \mu \mathrm{M}$ DNA primer were mixed and annealed at $95^{\circ} \mathrm{C}$ for $4 \mathrm{~min}$. Then the mixture was cooled to room temperature over $2 \mathrm{~h}$ and incubated with Phi29 DNA polymerase $(0.2 \mathrm{U} / \mu \mathrm{L})$, BSA $(0.4 \mu \mathrm{g} / \mu \mathrm{L})$ and $\mathrm{dNTPs}(0.1 \mathrm{mM})$ at $37^{\circ} \mathrm{C}$ for $5 \mathrm{~h}$ in $150 \mu \mathrm{L}$ of $1 \times$ Phi29 reaction buffer. Afterward, the mixture was incubated at $65^{\circ} \mathrm{C}$ for $10 \mathrm{~min}$ to denature the Phi29 
DNA polymerase, and purified by DNA purification kit to obtain the DNA nanowire.

Preparation of DNSL. H1 was synthesized by the equimolar mixing of tailed hairpin 1 with self-quenched signal and $\mathrm{H} 1$ connect at a final concentration of $10 \mu \mathrm{M}$ in PBS buffer. The connect contained a sequence complementary to the 24 base pairs at the 5'-end tail of hairpin 1, and another sequence with 24 bases complementary to the DNA nanowire for immobilization of hairpin 1. H2 was hairpin 2 tailed with 48 bases at 3'-end, half of them were used for its immobilization on the nanowire. After $\mathrm{H} 1$ and $\mathrm{H} 2$ were annealed in PBS buffer at $95{ }^{\circ} \mathrm{C}$ for $4 \mathrm{~min}$ and cooled to room temperature over $2 \mathrm{~h}$, the DNSL was prepared by mixing $25 \mu \mathrm{L}$ of $10 \mu \mathrm{M} \mathrm{H} 1,25 \mu \mathrm{L}$ of $10 \mu \mathrm{M} \mathrm{H} 2$ and $300 \mu \mathrm{L}$ of DNA nanowire for $2 \mathrm{~h}$ at $37{ }^{\circ} \mathrm{C}$, which led to the interval hybridization of $\mathrm{H} 1$ and $\mathrm{H} 2$ to DNA nanowire. Afterward, the formed DNSL was purified by ultrafiltration (100,000 MW cut-off membrane, Millipore) for three times. The concentration of H1 was used as DNSL concentration to simplify the comparison between DCR and HCR in homogeneous solution.

To measure the number of $\mathrm{H} 1$ and $\mathrm{H} 2$ in each DNSL, aminomethylcoumarin labelled H0 (AMCA-H0), 5-carboxyfluorescein labelled hairpin 1 without the quenching group (FAM-hairpin 1), and tetramethylrhodamine labelled H2 (TAMRA-H2) were selfassembled to form the tricolor DNSL, and the fluorescence intensities of three dyes in DNSL were measured and compared with the standard calibration curves of AMCAH0, FAM-hairpin1 and TAMRA-H2.

Electrophoresis Analysis. 8\% native polyacrylamide gel was prepared using 1×TBE buffer. The loading sample was prepared by mixing $7 \mu \mathrm{L}$ DNA sample, $1.5 \mu \mathrm{L}$ 
6×loading buffer and 1.5 $\mu \mathrm{L}$ UltraPowerTM dye, and placed for $3 \mathrm{~min}$ before injected into polyacrylamide gel. The gel electrophoresis was run at $90 \mathrm{~V}$ for $60 \mathrm{~min}$ in $1 \times \mathrm{TBE}$ buffer, and scanned with a Molecular Imager Gel Doc XR.

$0.6 \%$ agarose gel was prepared using $1 \times \mathrm{TBE}$ buffer. The gel electrophoresis was performed at $110 \mathrm{~V}$ for $60 \mathrm{~min}$ in $1 \times \mathrm{TBE}$ buffer, and visualized via a Molecular Imager Gel Doc XR.

Serum Stability Assay of DNSL. The solutions of $100 \mathrm{nM}$ DNSL and H1 were spiked with fetal bovine serum (FBS) respectively to a final concentration of $50 \mathrm{nM}$ in $10 \%$ FBS. Both solutions were incubated at $37^{\circ} \mathrm{C}$ for $12 \mathrm{~h}$, and the FAM fluorescence signals were measured every hour at $514 \mathrm{~nm}$ with $488 \mathrm{~nm}$ excitation.

In Vitro Detection of Target mRNA. $5 \mu \mathrm{L}$ survivin mRNA with various concentration were added in $50 \mu \mathrm{L}$ of $100 \mathrm{nM}$ DNSL solutions respectively, followed by incubation at $37^{\circ} \mathrm{C}$ for $15 \mathrm{~min}$. The resulting mixtures were immediately subjected to fluorescence measurements. Fluorescence spectra were recorded with excitation at $488 \mathrm{~nm}$. The slit width was set to be $5 \mathrm{~nm}$ for the excitation and $5 \mathrm{~nm}$ for the emission. For comparison of the amplification effects, the general HCR was performed by mixing $50 \mu \mathrm{L}$ of $100 \mathrm{nM} \mathrm{H} 1$ and $100 \mathrm{nM} \mathrm{H} 2$ with survivin mRNA under the same conditions.

Cell Culture. Human cervix carcinoma (HeLa) cells (KeyGEN Biotech, Nanjing, China) were cultured in Dulbecco's modified Eagle's medium (DMEM) supplemented with $10 \%$ fetal bovine serum (FBS), $100 \mu \mathrm{g} / \mathrm{mL}$ streptomycin and $100 \mathrm{U} / \mathrm{mL}$ penicillinstreptomycin at $37{ }^{\circ} \mathrm{C}$ in a humidified incubator containing $5 \% \mathrm{CO}_{2}$ and $95 \%$ air. Cell numbers were determined with a Petroff-Hausser cell counter (USA). 
MTT Assay. MTT assays were performed to investigate DNSL cytotoxicity. HeLa cells $\left(1 \times 10^{6}\right.$ cells/well $)$ were dispersed within replicate $96-$ well plates to a total volume of $200 \mu \mathrm{L} /$ well. Plates were maintained at $37{ }^{\circ} \mathrm{C}$ for $24 \mathrm{~h}$. After the medium was removed, the HeLa cells were washed twice with PBS and incubated with serial concentrations of the DNSL for $2 \mathrm{~h}$. Cells incubated with only the PBS was served as control. The cells were washed twice with PBS buffer in the following and $50 \mu \mathrm{L}$ of 5 $\mathrm{mg} / \mathrm{mL}$ MTT solution was added and incubated for $4 \mathrm{~h}$. After removing the remaining MTT solution, $150 \mu \mathrm{L}$ of dimethylsulphoxide was added to dissolve the formazan crystals precipitates. After shaking the plate for $15 \mathrm{~min}$, the optical density at a wavelength of $490 \mathrm{~nm}$ was measured with a Bio-Rad microplate reader.

Confocal Fluorescence Imaging and Flow Cytometric Assay. $1 \times 10^{4} \mathrm{HeLa}$ cells were seeded in a confocal dish for $24 \mathrm{~h}$ at $37^{\circ} \mathrm{C}$, then incubated with $200 \mu \mathrm{L}$ culture medium containing $100 \mathrm{nM}$ DNSL for $2 \mathrm{~h}$ at $37{ }^{\circ} \mathrm{C}$. After washing twice with PBS, the fluorescence of cells was visualized from 510 to $550 \mathrm{~nm}$ with the excitation wavelength of $488 \mathrm{~nm}$ for FAM. All images were digitized and analyzed with Leica Application Suite Advanced Fluorescence (LAS-AF) software package. The fluorescence intensity in the cell area for each image was determined with Adobe Photoshop software. The flow cytometric assay was performed in PBS with FL1 channel.

The survivin mRNA inhibited experiment was performed as follows: HeLa cells were seeded in a confocal dish for $24-\mathrm{h}$ incubation, and $200 \mu \mathrm{L}$ culture medium containing YM155 as an inhibitor at a given concentration was then added into each cell-adhered dish. After incubation for $48 \mathrm{~h}$ at $37^{\circ} \mathrm{C}$, the cells were washed with PBS and incubated 
with $200 \mu \mathrm{L}$ culture medium containing $100 \mathrm{nM} \mathrm{DNSL}$ for $2 \mathrm{~h}$ at $37^{\circ} \mathrm{C}$ to perform confocal fluorescence imaging.

RT-PCR Analysis of survivin mRNA in Cells. Total RNAs from HeLa cells were extracted using Trizol reagent (Invitrogen, USA), and cDNA was prepared using PrimeScriptRT reagent kit (Takara), which was detected with real-time PCR (RT-PCR) to calculate intracellular survivin mRNA level. All data were evaluated with respect to the mRNA expression by normalizing to the expression of actin and using the $2^{-\Delta \Delta \mathrm{Ct}}$ method. The primers used in this experiment were: survivin forward, 5'-TCCACTGC CCCACTGAGAAC-3'; survivin reverse, 5'-TGGCTCCCAGCCTTCCA-3'; actin forward, 5'-AAAGACCTGTACGCCAACACAGTGCTGTCTGG-3'; actin reverse, 5'-CGTCATACTCCTGCTTGCT GATCCACATCTGC-3'.

\section{ASSOCIATED CONTENT}

The authors declare no competing financial interest.

\section{Supporting Information}

The Supporting Information is available free of charge on the ACS Publications website at DOI: http://pubs.acs.org.

Serum stability of H1 in DNSL; responses of DNSL, control DNSL, and H1/H2 mixture to target mRNA; responses of DNSL to different mRNAs; time course and Z-stack confocal images of HeLa cells incubated with DNSL; confocal fluorescence images of HeLa cells incubated with various control DNSLs and control HeLa cells incubated with DNSL; cytotoxicity of DNSL to HeLa cells; quantitative analysis of mRNA in HeLa cells via flow cytometry and RT-PCR; 
simultaneous confocal fluorescence imaging of s-mRNA and T-mRNA in HeLa cells; sequences of oligonucleotides (PDF)

\section{AUTHOR INFORMATION}

\section{Corresponding Authors}

*E-mail: yingliu@nju.edu.cn; hxju@nju.edu.cn

\section{ACKNOWLEDGMENTS}

We gratefully acknowledge National Natural Science Foundation of China (21605083, 21635005, 21361162002), Natural Science Foundation of Jiangsu Province (BK20160644), and the National Research Foundation for Thousand Youth Talents Plan of China.

\section{REFERENCES}

(1) Zhao, Y. X.; Chen, F.; Li, Q.; Wang, L. H.; Fan, C. H. Isothermal Amplification of Nucleic Acids. Chem. Rev. 2015, 115, 12491-12545.

(2) Larsson, C.; Grundberg, I.; Söderberg, O.; Nilsson, M. In Situ Detection and Genotyping of Individual mRNA Molecules. Nat. Methods 2010, 7, 395-397.

(3) Qian, L. L.; Winfree, E. Scaling Up Digital Circuit Computation with DNA Strand Displacement Cascades. Science 2011, 332, 1196-1201.

(4) Zhang, D. Y.; Turberfield, A. J.; Yurke, B.; Winfree, E. Engineering EntropyDriven Reactions and Networks Catalyzed by DNA. Science 2007, 318, 1121-1125.

(5) Ali, M. M.; Li, F.; Zhang, Z. Q.; Zhang, K. X.; Kang, D. K.; Ankrum, J. A.; Le, 
X. C.; Zhao, W. A. Rolling Circle Amplification: a Versatile Tool for Chemical Biology, Materials Science and Medicine. Chem. Soc. Rev. 2014, 43, 3324-3341.

(6) Deng, R. J.; Tang, L. H.; Tian, Q. Q.; Wang, Y.; Lin, L.; Li, J. H. Toehold-Initiated Rolling Circle Amplification for Visualizing Individual MicroRNAs in Situ in Single Cells. Angew. Chem. Int. Ed. 2014, 53, 2389-2393.

(7) Ge, J.; Zhang, L. L.; Liu, S. J.; Yu, R. Q.; Chu, X. A Highly Sensitive TargetPrimed Rolling Circle Amplification (TPRCA) Method for Fluorescent in Situ Hybridization Detection of microRNA in Tumor Cells. Anal. Chem. 2014, 86, $1808-1815$.

(8) Wu, C. C.; Cansiz, S.; Zhang, L. Q.; Teng, I. T.; Qiu, L. P.; Li, J.; Liu, Y.; Zhou, C. S.; Hu, R.; Zhang, T.; Cui, C.; Cui, L.; Tan, W. H. A Nonenzymatic Hairpin DNA Cascade Reaction Provides High Signal Gain of mRNA Imaging Inside Live Cells. $J$. Am. Chem. Soc. 2015, 137, 4900-4903.

(9) Dirks, R. M.; Pierce, N. A. Triggered Amplification by Hybridization Chain Reaction. Proc. Natl. Acad. Sci. USA 2004, 101, 15275-15278.

(10) Yin, P.; Choi, H. M. T.; Calvert, C. R.; Pierce, N. A. Programming Biomolecular Self-Assembly Pathways. Nature 2008, 451, 318-322.

(11) Wang, F.; Lu, C. H.; Willner, I. From Cascaded Catalytic Nucleic Acids to Enzyme-DNA Nanostructures: Controlling Reactivity, Sensing, Logic Operations, and Assembly of Complex Structures. Chem. Rev. 2014, 114, 2881-2941.

(12) Choi, H. M. T.; Beck, V. A.; Pierce, N. A. Next-Generation in Situ Hybridization Chain Reaction: Higher Gain, Lower Cost, Greater Durability. ACS Nano 2014, 8, 
$4284-4294$.

(13) Li, B. L.; Jiang, Y.; Chen, X.; Ellington, A. D. Probing Spatial Organization of DNA Strands Using Enzyme-Free Hairpin Assembly Circuits. J. Am. Chem. Soc. 2012, $134,13918-13921$.

(14) Koos, B.; Cane, G.; Grannas, K.; Lof, L.; Arngården, L.; Heldin, J.; Clausson, C.; Klaesson, A.; Hirvonen, M. K.; de Oliveira, F. M. S.; Talibov, V. O.; Pham, N. T.; Auer, M.; Danielson, H.; Haybaeck, J.; Kamali-Moghaddam, M.; Soderberg, O. Proximity-Dependent Initiation of Hybridization Chain Reaction. Nat. Commun. 2015, $6,7294$.

(15) Wu, Z.; Liu, G. Q.; Yang, X. L.; Jiang, J. H. Electrostatic Nucleic Acid Nanoassembly Enables Hybridization Chain Reaction in Living Cells for Ultrasensitive mRNA Imaging. J. Am. Chem. Soc. 2015, 137, 6829-6836.

(16) Li, L.; Feng, J.; Liu, H. Y.; Li, Q. L.; Tong, L. L.; Tang, B. Two-Color Imaging of MicroRNA with Enzyme-Free Signal Amplification via Hybridization Chain Reactions in Living Cells. Chem. Sci. 2016, 7, 1940-1945.

(17) Jiang, Y. S.; Li, B. L.; Milligan, J. N.; Bhadra, S.; Ellington, A. D. Real-Time Detection of Isothermal Amplification Reactions with Thermostable Catalytic Hairpin Assembly. J. Am. Chem. Soc. 2013, 135, 7430-7433.

(18) Jiang, Y. S.; Bhadra, S.; Li, B. L.; Ellington, A. D. Mismatches Improve the Performance of Strand-Displacement Nucleic Acid Circuits. Angew. Chem. Int. Ed. 2014, 53, 1845-1848.

(19) Bi, S.; Yue, S. Z.; Zhang, S. S. Hybridization Chain Reaction: a Versatile 
Molecular Tool for Biosensing, Bioimaging, and Biomedicine. Chem. Soc. Rev. 2017, 46, 4281-4298.

(20) Jung, C.; Ellington, A. D. Diagnostic Applications of Nucleic Acid Circuits. Acc. Chem. Res. 2014, 47, 1825-1835.

(21) Agapakis, C. M.; Boyle, P. M.; Silver, P. Natural Strategies for the Spatial Optimization of Metabolism in Synthetic Biology. Nat. Chem. Biol. 2012, 8, 527-535.

(22) Lee, J. W.; Na, D.; Park, J. M.; Lee, J.; Choi, S.; Lee, S. Y. Systems Metabolic Engineering of Microorganisms for Natural and Non-Natural Chemicals. Nat. Chem. Biol. 2012, 8, 536-546.

(23) Zhao, Z.; Fu, J. L.; Dhakal, S.; Buck, A. J.; Liu, M. H.; Zhang, T.; Woodbury, N. W.; Liu, Y.; Walter, N. G.; Yan, H. Nanocaged Enzymes with Enhanced Catalytic Activity and Increased Stability against Protease Digestion. Nat. Commun. 2016, 7, 10619.

(24) Ke, G. G.; Liu, M. H.; Jiang, S. X.; Qi, X. D.; Yang, Y. H. R.; Wootten, S.; Zhang, F.; Zhu, Z.; Liu, Y.; Yang, C. Y. J.; Yan, H. Directional Regulation of Enzyme Pathways through the Control of Substrate Channeling on a DNA Origami Scaffold. Angew. Chem. Int. Ed. 2016, 55, 7483-7486.

(25) Wilner, O. I.; Weizmann, Y.; Gill, R.; Lioubashevski, O.; Freeman, R.; Willner, I. Enzyme Cascades Activated on Topologically Programmed DNA Scaffolds. Nat. Biotechnol. 2009, 4, 249-254.

(26) Chatterjee, G.; Dalchau, N.; Muscat, R. A.; Phillips, A.; Seelig, G. A Spatially Localized Architecture for Fast and Modular DNA Computing. Nat. Nanotechnol. 2017, 
12, 1-10.

(27) Kopperger, E.; Pirzer, T.; Simmel, F. C. Diffusive Transport of Molecular Cargo Tethered to a DNA Origami Platform. Nano Lett. 2015, 15, 2693-2699.

(28) Bui, H.; Miao, V.; Garg, S.; Mokhtar, R.; Song, T. Q.; Reif, J. Design and Analysis of Localized DNA Hybridization Chain Reactions. Small 2017, 1602983.

(29) Teichmann, M.; Kopperger, E.; Simmel, F. C. Robustness of Localized DNA Strand Displacement Cascades. ACS Nano 2014, 8, 8487-8496.

(30) Choi, H. M. T.; Chang, J. Y.; Trinh, L. A.; Padilla, J. E.; Fraser, S. E.; Pierce, N. A. Programmable in Situ Amplification for Multiplexed Imaging of mRNA Expression. Nat. Biotechnol. 2010, 28, 1208-1212.

(31) He, X. W.; Zeng, T.; Li, Z.; Wang, G. L.; Ma, N. Catalytic Molecular Imaging of MicroRNA in Living Cells by DNA Programmed Nanoparticle Disassembly. Angew. Chem. Int. Ed. 2016, 55, 3073-3076.

(32) Lv, Y. F.; Hu, R.; Zhu, G. Z.; Zhang, X. B.; Mei, L.; Liu, Q. L.; Qiu, L. P.; Wu, C. C.; Tan, W. H. Preparation and Biomedical Applications of Programmable and Multifunctional DNA Nanoflowers. Nat. Protoc. 2014, 86, 1808-1815.

(33) Lau, K. L.; Hamblin, G. D.; Sleiman, H. F. Gold Nanoparticle 3D-DNA Building Blocks: High Purity Preparation and Use for Modular Access to Nanoparticle Assemblies. Small 2014, 10, 660-666.

(34) Tay, C. Y.; Yuan, L.; Leong, D. T. Nature-Inspired DNA Nanosensor for RealTime in Situ Detection of mRNA in Living Cells. ACS Nano 2015, 9, 5609-5617.

(35) Xu, L. P.; Chen, Y. X.; Yang, G.; Shi, W. X.; Dai, B.; Li, G. N.; Cao, Y. H.; Wen, 
Y. Q.; Zhang, X. J.; Wang, S. T. Ultratrace DNA Detection Based on the CondensingEnrichment Effect of Superwettable Microchips. Adv. Mater. 2015, 27, 6878-6884.

(36) Zhang, H. Q.; Li, F.; Dever, B.; Wang, C.; Li, X. F.; Le, X. C. Assembling DNA through Affinity Binding to Achieve Ultrasensitive Protein Detection. Angew. Chem. Int. Ed. 2013, 52, 10698-10705.

(37) Wu, P. W.; Hwang, K.; Lan, T.; Lu, Y. A DNAzyme-Gold Nanoparticle Probe for Uranyl Ion in Living Cells. J. Am. Chem. Soc. 2013, 135, 5254-5257.

(38) Yamanaka, K.; Nakahara, T.; Yamauchi, T.; Kita, A.; Takeuchi, M.; Kiyonaga, F.; Kaneko, N.; Sasamata, M. Antitumor Activity of YM155, a Selective SmallMolecule Survivin Suppressant, alone and in Combination with Docetaxel in Human Malignant Melanoma Models. Clin. Cancer Res. 2011, 17, 5423-5431.

(39) Yang, Y. J.; Huang, J.; Yang, X. H.; Quan, K.; Wang, H.; Ying, L.; Xie, N. L.; Ou, M.; Wang, K. M. FRET Nanoflares for Intracellular mRNA Detection: Avoiding False Positive Signals and Minimizing Effects of System Fluctuations. J. Am. Chem. Soc. 2015, 137, 8340-8343.

(40) He, D. G.; He, X.; Yang, X.; Li, H. W. A Smart ZnO@polydopamine-Pucleic Acid Nanosystem for Ultrasensitive Live Cell mRNA Imaging by the Target-Triggered Intracellular Self-Assembly of Active DNAzyme Nanostructures. Chem. Sci. 2017, 8, $2832-2840$.

(41) Han, D.; Wu, C. C.; You, M. X.; Zhang, T.; Wan, S.; Chen, T.; Qiu, L. P.; Zheng, Z.; Liang, H.; Tan, W. H. A Cascade Reaction Network Mimicking the Basic Functional Steps of Adaptive Immune Response. Nat. Chem. 2015, 7, 835-841. 
(42) Kim, E. J.; Zwi-Dantsis, L.; Reznikov, N.; Hansel, C. S.; Agarwal, S.; Stevens, M. M. One-Pot Synthesis of Multiple Protein-Encapsulated DNA Flowers and Their Application in Intracellular Protein Delivery. Adv. Mater. 2017, 29, 1701086. 


\section{FIGURE CAPTIONS}

Scheme 1. Schematic illustration of (a) DNSL synthesis based on interval hybridization of $\mathrm{H} 1$ and $\mathrm{H} 2$ to a DNA nanowire, and (b) targeted delivery of DNSL and imaging of target mRNA in living cells based on accelerated DCR along DNA nanowire.

Figure 1. (a) PAGE analysis of DNSL self-assembly. Lanes 1-5 represent DNA nanowire, H1, H2, DNSL, and DNA ladder marker, respectively. (b) AFM phase image of DNSL. (c) Cross-section profile of the white line in (b).

Figure 2. (a) Time-dependent fluorescence spectra of DCR in $100 \mathrm{nM}$ DNSL, and HCR in a homogeneous solution containing $100 \mathrm{nM} \mathrm{H} 1$ and $100 \mathrm{nM} \mathrm{H} 2$ in response to 10 $\mathrm{nM}$ survivin mRNA ( $s$-mRNA). (b) Comparison of the reaction area and local concentration of $\mathrm{H} 1$ and $\mathrm{H} 2$ for DCR and HCR.

Figure 3. Schematic and fluorescence spectra of (a, b) DCR in DNSL, (d, e) HCR in homogeneous solution, and (g, h) H1R in response to survivin mRNA (s-mRNA) at various concentrations, and (c, $\mathrm{f}$ and $\mathrm{i}$ ) their corresponding calibration curves. The dotted horizontal lines indicate the fluorescence intensity for LOD estimation. The data error bars indicate means $\pm \operatorname{SD}(n=3)$.

Figure 4. (a) Confocal fluorescence images of HeLa cells incubated with DNSL, mixture of $\mathrm{H} 1$ and $\mathrm{H} 2$, and $\mathrm{H} 1$. The scale bar indicates $20 \mu \mathrm{m}$. (b) Fluorescence intensities obtained from (a). The data error bars indicate means $\pm \operatorname{SD}(n=3)$.

Figure 5. (a) Confocal fluorescence images of HeLa cells treated with various concentrations of YM155 followed by incubation with DNSL or mixture of H1 and H2. The scale bar indicates $20 \mu \mathrm{m}$. Fluorescence intensities for (b) mixture of $\mathrm{H} 1$ and $\mathrm{H} 2$, 
and (c) DNSL incubated HeLa cells. The data error bars indicate means \pm SD $(n=3)$. 
Scheme 1

a)

Circular DNA

template
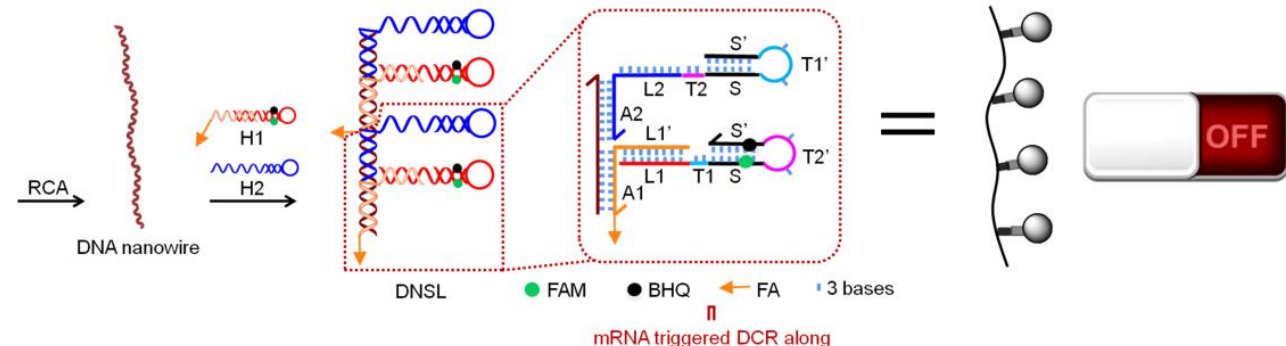

b) 8 snom

sinnos

M. M.
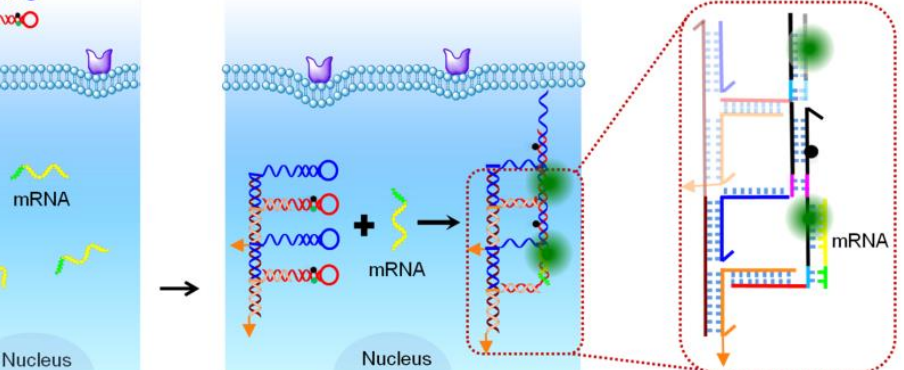

$=:$

Nucleus

Nucleus

DNA nanowire 
Figure 1
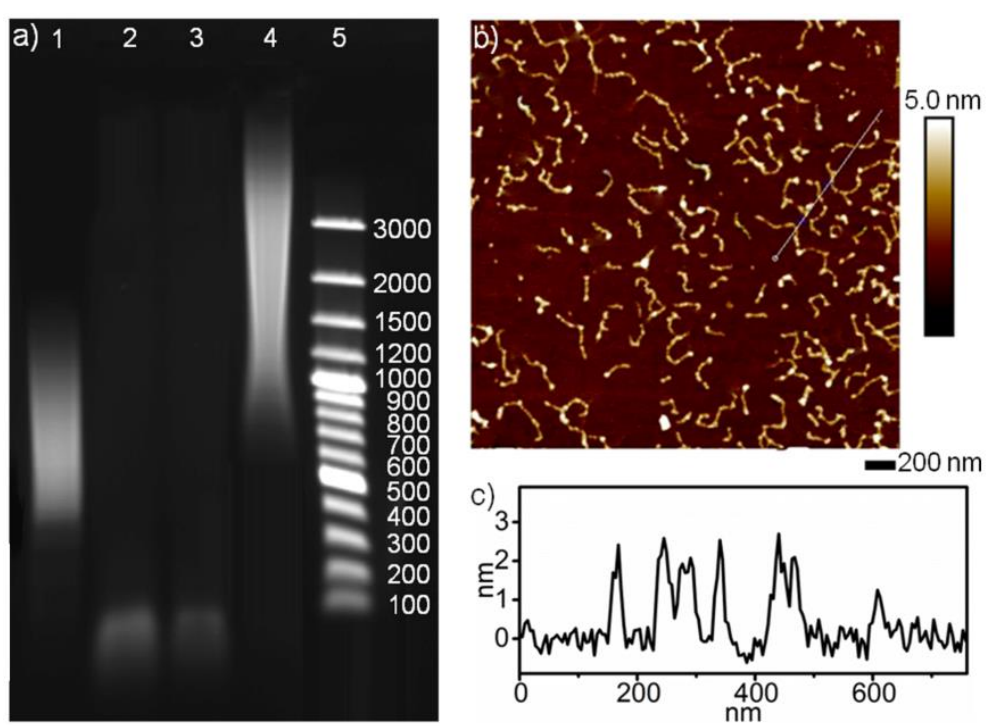

c) 3

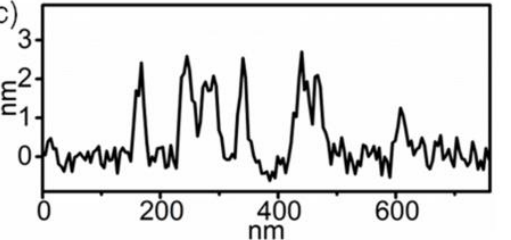


Figure 2
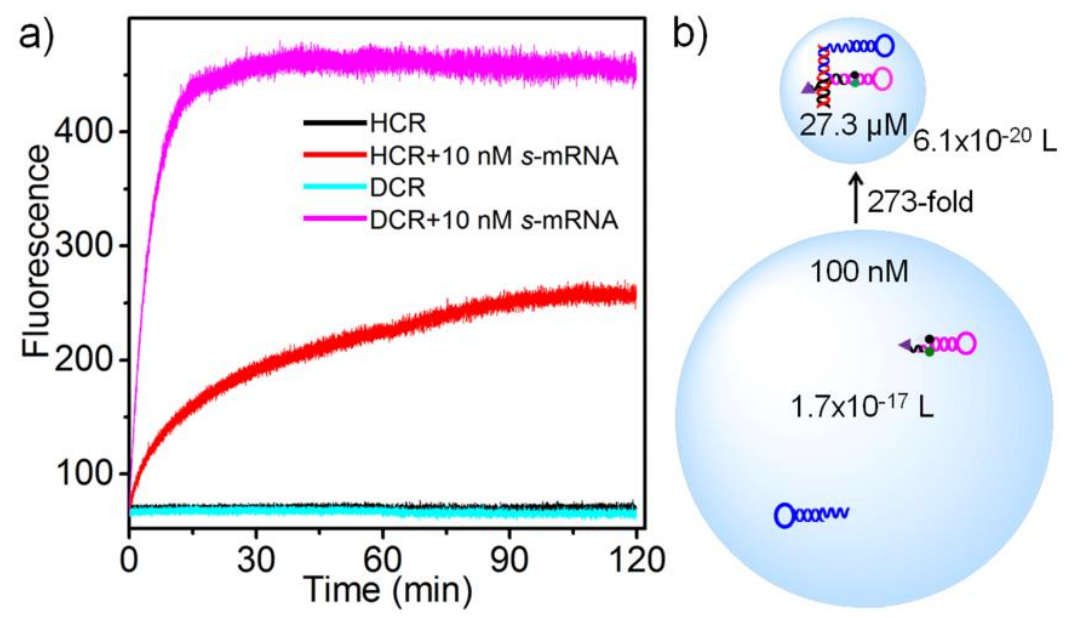
Figure 3

a)

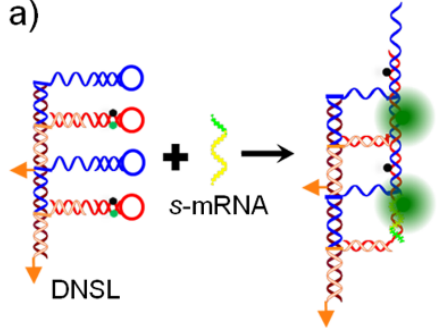

d)

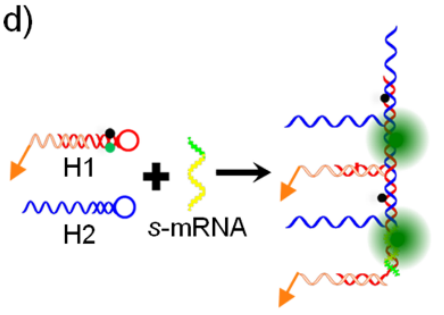

g)

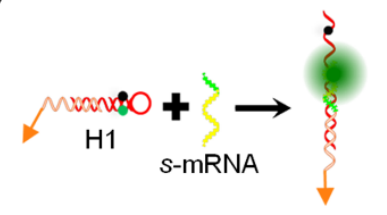

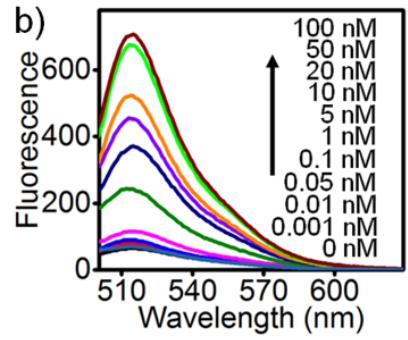
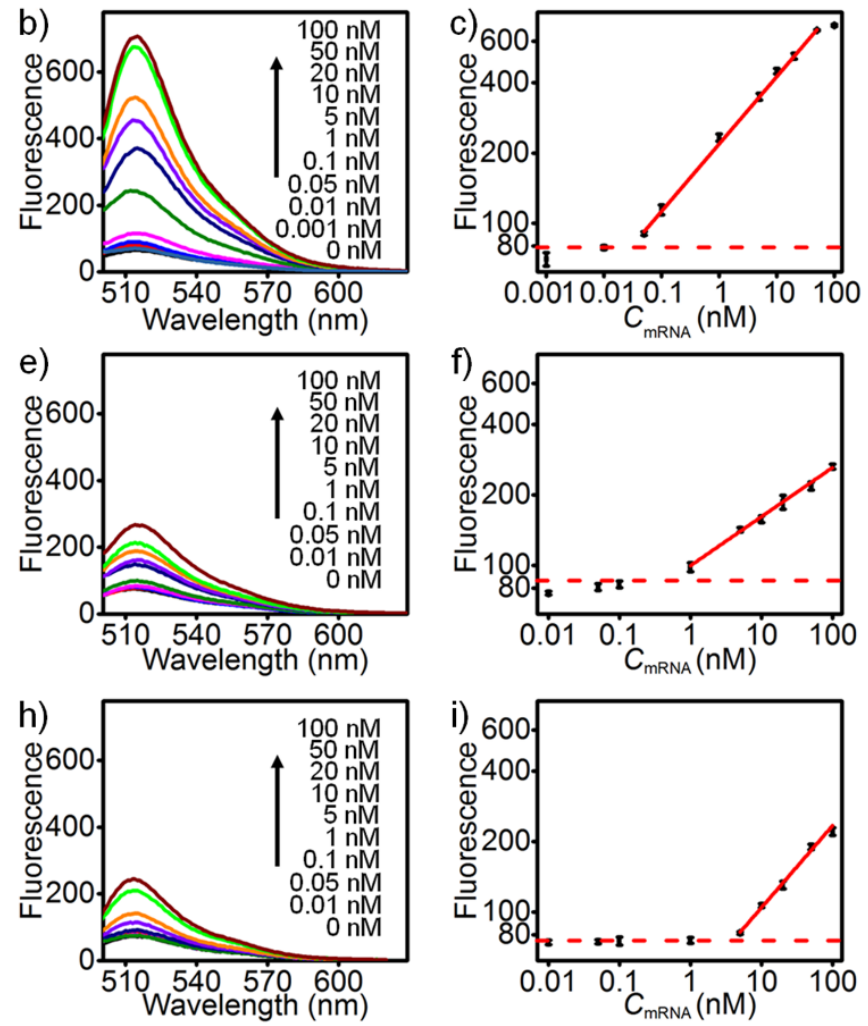
Figure 4
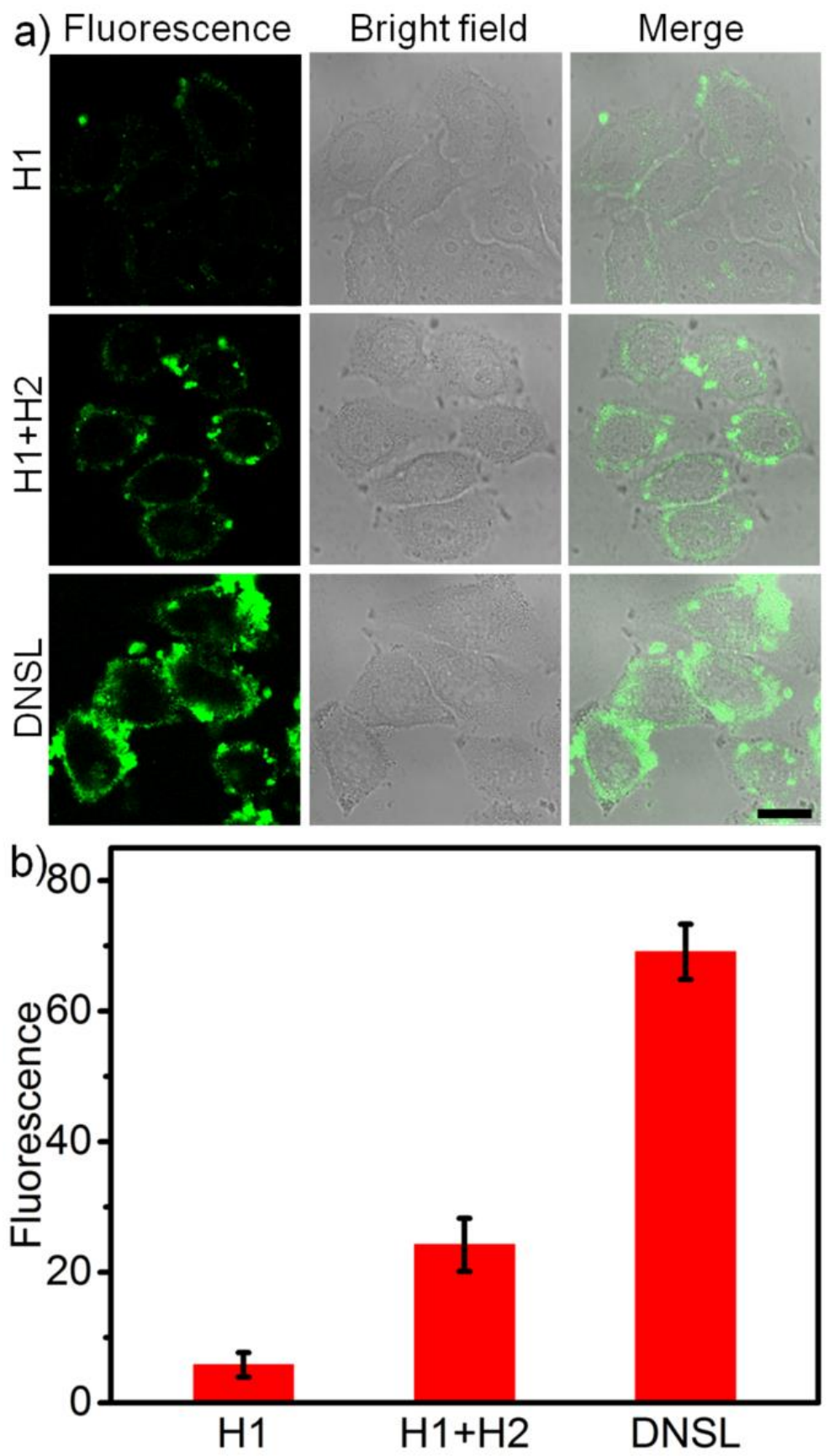
Figure 5
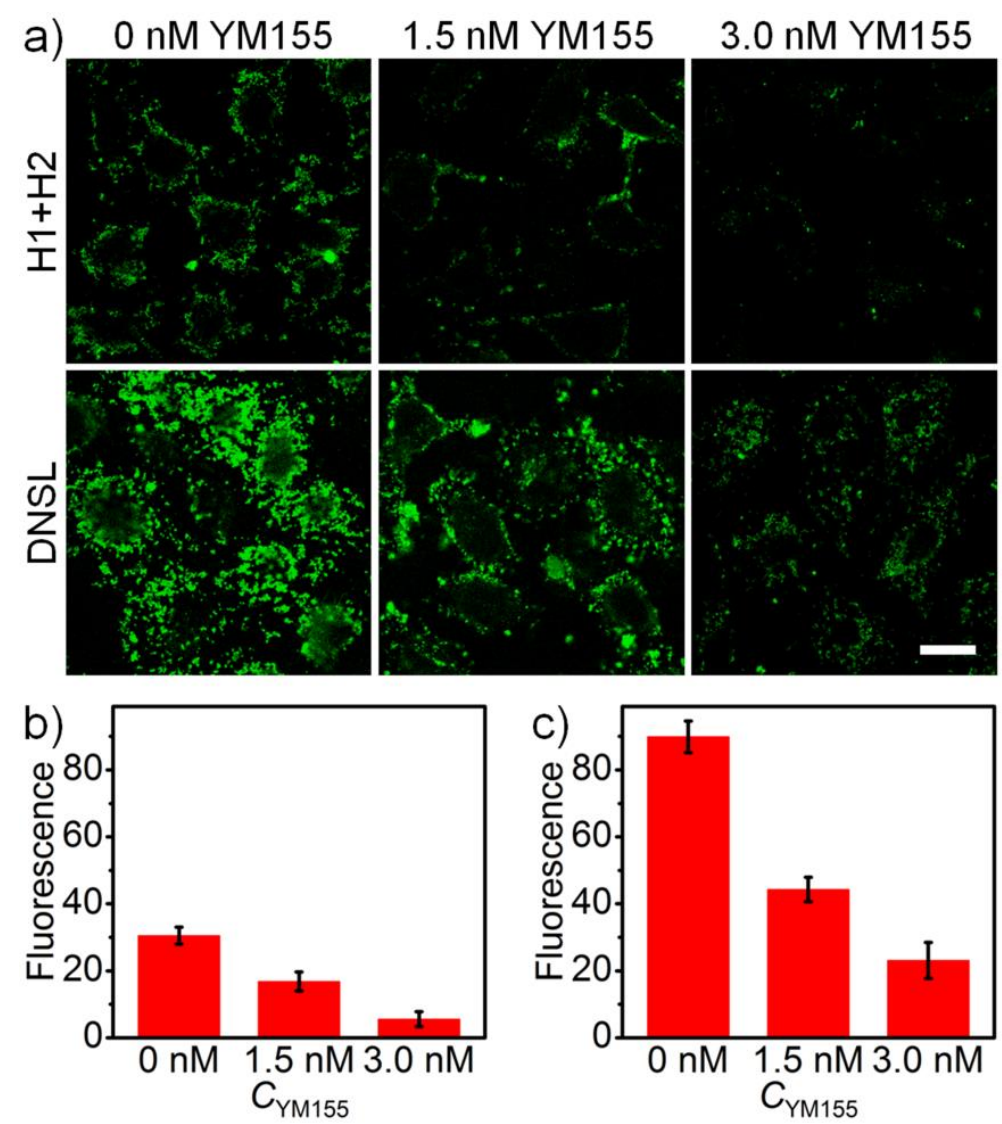


\section{For TOC only}

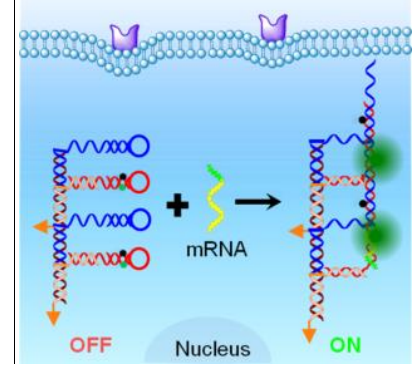

